\title{
Efficacy of electro-acupuncture and manual acupuncture versus sham acupuncture for knee osteoarthritis: statistical analysis plan for a randomized controlled trial
}

\author{
Jian-Feng Tu' ${ }^{1}$ Jing-Wen Yang ${ }^{1}$, Li-Qiong Wang ${ }^{1}$, Yang Wang ${ }^{2}$, Jin-ling Li', Na Zhang ${ }^{1}$, Lu-Lu Lin ${ }^{1}$,
} Zhang-Sheng $\mathrm{Yu}^{3,4}$ and Cun-Zhi Liu ${ }^{1 *}$ (D)

\begin{abstract}
Background: Acupuncture is widely used for knee osteoarthritis (KOA), despite contradictory evidence. This study is designed to determine the efficacy of electro-acupuncture and manual acupuncture versus sham acupuncture for KOA.

Methods/design: This is a multi-center three-arm randomized controlled trial. It will enroll 480 participants with KOA in China. Participants will be randomly assigned 1:1:1 to receive 24 sessions of electro-acupuncture, manual acupuncture, or sham acupuncture over 8 weeks. The primary outcome is the response rate, which is the proportion of patients who achieve the minimal clinically important improvement in pain and function at 8 weeks. The primary outcome will be analyzed using the Z-test with the intention-to-treat set. Secondary outcomes include pain, function, global patient assessment, and quality of life. Full details of the statistical analysis plan for the primary and secondary outcomes will be described in this article. The statistical analysis plan was written and submitted without knowledge of the study data.
\end{abstract}

Discussion: The data will be analyzed according to this pre-specified statistical analysis plan to avoid data-driven analysis and to enhance the transparency of the trial. The aim of the trial is to provide high-quality evidence on the efficacy of acupuncture for KOA.

Trial registration: Clinicaltrials.gov, NCT03366363. Registered on 20 November 2017.

Keywords: Acupuncture, Knee osteoarthritis, Statistical analysis plan

\section{Background}

Knee osteoarthritis (KOA) is one of the leading causes of chronic pain and disability in older adults [1], with symptomatic KOA affecting $8.1 \%$ of Chinese people [2] and $1.6-14.9 \%$ of Europeans according to age class [3]. The socioeconomic burden of KOA is large, amounting to between $1.0 \%$ and $2.5 \%$ of the gross domestic product in developed countries [4].

Since no disease-modifying treatment is available, the current management of KOA is symptomatic [5], often with non-steroid anti-inflammatory drugs. However, these

\footnotetext{
* Correspondence: Icz623780@126.com

${ }^{1}$ School of Acupuncture-Moxibustion and Tuina, Beijing University of Chinese Medicine, 11 Beisanhuan East Road, Chaoyang District, Beijing 100029, China Full list of author information is available at the end of the article
}

have a limited effect $[6,7]$. Although total knee replacement is an effective treatment for symptomatic end-stage disease, approximately $15 \%$ of patients have continuing pain and mobility problems after surgery and the lifespan of prostheses is limited [8].

Acupuncture is increasingly used in clinical practice [9], although evidence of its efficacy is contradictory $[10,11]$. Acupuncture has a dose-effect relationship [12]. However, the dose of acupuncture administered in several previous trials was far from adequate [13]. The frequency of acupuncture is one of the key factors of a dose [14]. A review suggested that the frequency of acupuncture is usually three to five sessions per week in China, whereas it is mostly one session per week in Europe and America [15]. Based on our previous pilot trial [16], high-dose acupuncture (24 
sessions in 8 weeks) may be an effective option for KOA. Electro-acupuncture (EA) combines manual acupuncture (MA) with an electric stimulus [17]. Both EA and MA are frequently used in clinical practice. Therefore, the current trial is designed to evaluate the effect of EA and MA, compared with sham acupuncture (SA), in patients with KOA.

The protocol of the trial has been published previously [18] and provides more detail on the trial rationale, eligibility criteria, and interventions. This article aims to report in detail the statistical analysis plan to reduce the risks of reporting bias and enhance the transparency of the trial. The statistical analysis plan was approved on 30 October 2017 (version 1.0) and drafted without knowledge of any of the results.

\section{Methods/design}

\section{Study design}

This three-arm randomized sham-controlled trial has been approved by the ethics committees at each of the nine participating hospitals. Eligible KOA participants, diagnosed according to the American College of Rheumatology criteria [19], are randomly assigned (1:1:1) to receive 24 sessions of EA, MA, or SA over 8 weeks. The block randomization, with block sizes of 6 and 9, is stratified by study center and is performed via a web-based randomization system. Superficial insertion at nonacupoints with no electric current will be used for the SA group, which is one of the most commonly used approaches for administering sham treatments in acupuncture trials. The nature of acupuncture means that acupuncturists are not blinded to treatment allocation; however, patients, outcome assessors, and statisticians will remain masked. Informed consent will be obtained from each participant before randomization. The trial has been registered with ClinicalTrials.gov (NCT03366363).

\section{Objectives}

The objective of the current study is to determine if EA or MA improve clinical outcomes at 8 weeks in patients with KOA. The following two null hypotheses will be tested:

H1: There is no difference in the patients' response rate between the EA group and the SA group.

$\mathrm{H} 2$ : There is no difference in the patients' response rate between the MA group and the SA group.

\section{Outcomes}

\section{Primary outcome}

The primary outcome is the response rate [20], which is the proportion of patients who achieve the minimal clinically important improvement in pain and function at 8 weeks post-randomization. The average pain over the previous week will be assessed using an 11-point numerical rating scale (NRS) [21] with scores ranging from 0 to 10.
The minimal clinically important improvement in pain is defined as 2 points in the NRS $[11,22]$. The average function over the previous week is measured using the Western Ontario and McMaster Universities Osteoarthritis Index (WOMAC) function subscale [23] with scores ranging from 0 to 68 . The minimal clinically important improvement in function is defined as 6 points in the WOMAC function subscale [11, 22]. The criteria for a participant to be a responder are presented in Fig. 1. The response rate is also measured at weeks 4,16 , and 26 after randomization.

\section{Secondary outcomes}

The secondary outcomes are:

- Numerical rating scale [21]: an 11-point patientreported outcome measure (PROM) with scores ranging from 0 (no pain) to 10 (worst pain)

- WOMAC [23] pain subscale: a 5-item PROM with total scores ranging from 0 to 20; higher scores indicate worse pain

- WOMAC [23] function subscale: a 17-item PROM with total scores ranging from 0 to 68; lower scores indicate better physical function

- WOMAC [23] stiffness subscale: a 2-item PROM with total scores ranging from 0 to 8 ; higher scores indicate more stiffness

- Patient global assessment [24]: a 5-point Likert scale

- 12-item Short Form Health Survey (SF-12) [25] physical dimension: total scores range from 0 to 100; lower scores indicate a worse quality of life

- SF-12 [25] mental dimension: total scores range from 0 to 100; higher scores indicate a better quality of life

For the patient global assessment, participants are asked how their knee symptoms were during the past week. Answers can include "extremely improved," "slightly improved," "not changed," "slightly aggravated," and "extremely aggravated."

The NRS, WOMAC, patient global assessment, and SF-12 are measured at $4,8,16$, and 26 weeks after randomization. The blinding assessment is measured at 4 and 8 weeks after randomization. The credibility and expectancy of participants are measured $5 \mathrm{~min}$ after the first acupuncture session [26]. The use of rescue medicine is also recorded throughout the trial.

\section{Safety outcome}

Adverse events are recorded throughout the trial. Based on the potential relationship between acupuncture and adverse events, adverse events are categorized as related to the treatment or not. 
Absolute change in WOMAC function subscale $\geq 6$ points

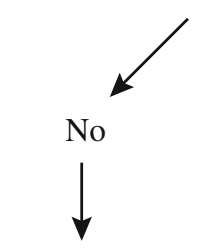

No Response

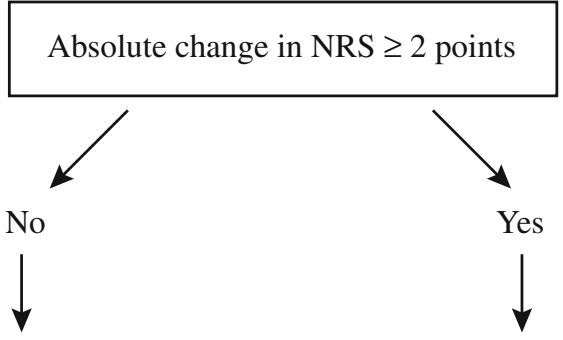

Response

Fig. 1 Responder criteria. WOMAC Western Ontario and McMaster Universities Osteoarthritis Index

\section{Sample size}

Based on the results of our previous trial [16], the response rates for the EA, MA, and SA groups were assumed to be $70 \%, 60 \%$, and $40 \%$, respectively. With a twosided significance level of $2.5 \%$ and power of $80 \%, 128$ participants in each group will be required to detect a difference as small as $20 \%$ between each acupuncture group and the control group. The two-sided significance level of $2.5 \%$ is a Bonferroni-adjusted alpha level as per the two predefined primary comparisons: EA vs. SA and MA vs. SA. With an estimated loss-to-follow-up rate of $20 \%, 480$ participants will be recruited in total.

\section{Statistical analysis Statistical analysis population}

A modified full analysis set, a per-protocol set, and a safety set will be used in this trial. The modified full analysis set will consist of all randomized participants who have at least one post-baseline measurement according to the modified intention-to-treat principle. The modified full analysis set will be the primary analysis set, and all analyses will be conducted for this population if not otherwise stated. Analyses of the modified full analysis set will provide an estimate of the effects of EA and MA.

The per-protocol set will include those who complete the treatment and follow-up on time according to the protocol without major violations. Major violations of the protocol will be judged during the blinded audit of the data. They include but are not limited to not meeting the inclusion criteria, meeting the exclusion criteria, receiving other treatments that might affect the symptoms of KOA during the trial, and completing $<20$ sessions of acupuncture. The per-protocol set will be the secondary analysis set and will be used for the sensitivity analyses.

All those who are randomized and have received at least one session of acupuncture will be defined as the safety set, which is used for the safety analyses.

\section{General analysis principles}

All data will be summarized by treatment group. Numbers (percentages) will be used to describe categorical data. Either means (standard deviations) or medians (interquartile ranges) will be used for quantitative data depending on whether the variables are normally distributed. If not otherwise stated, the significance level will be set at 0.05 . The Bonferroni method will be used to adjust the significance level for multiple comparisons for the primary outcome. The conclusions will be based on the analysis of the primary outcome, and all secondary outcomes will be analyzed to support the primary analysis. All analyses will be carried out using SAS 9.3 (Cary, NC).

\section{Descriptive analyses}

The numbers of participants screened, excluded, randomly assigned to each group, interviewed at each follow-up, and analyzed will be summarized using a flow diagram recommended by CONSORT [27] (Fig. 2) . Reasons for the losses to follow-up and withdrawals will also be listed by treatment arm. 


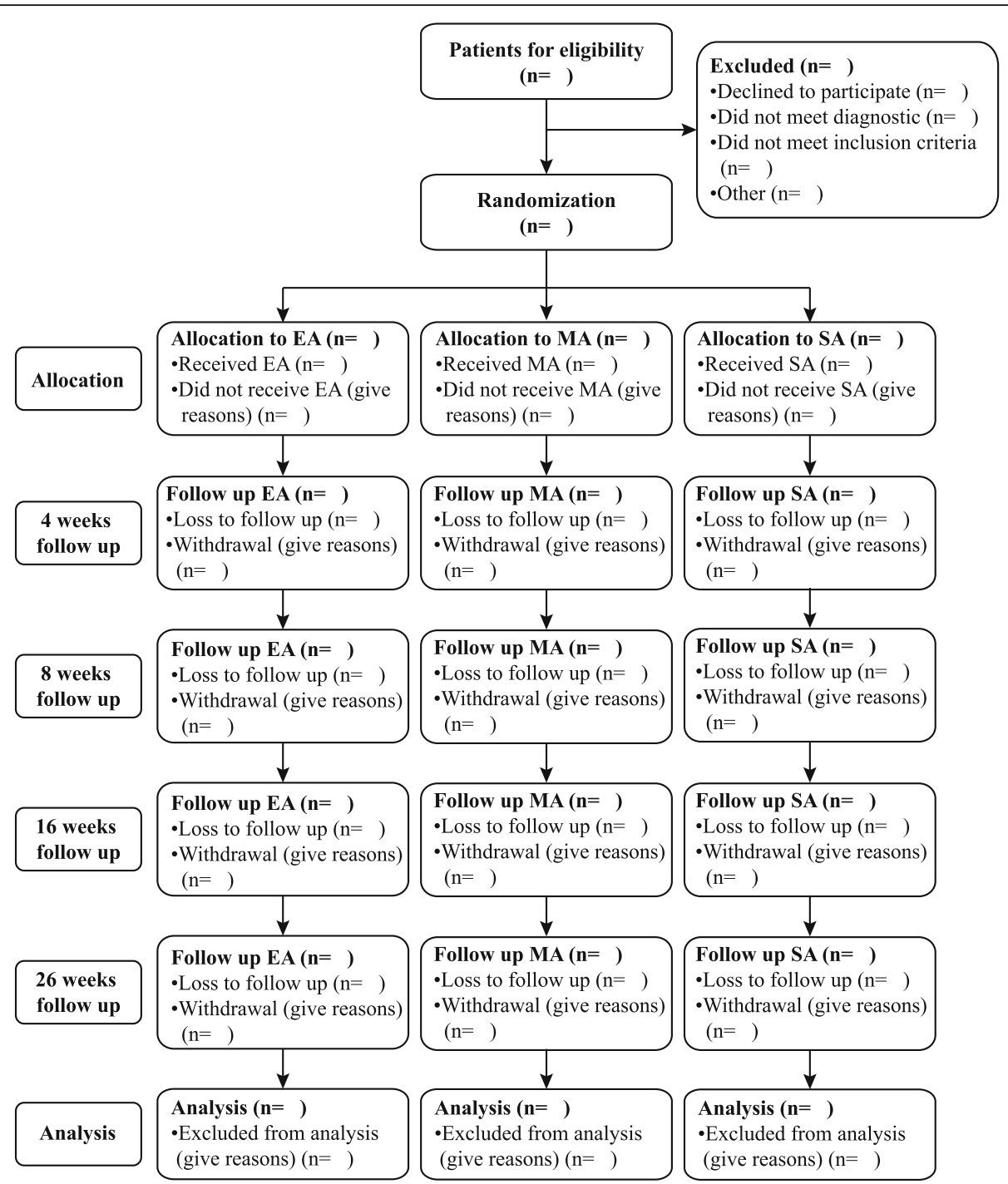

Fig. 2 Flow diagram. EA electro-acupuncture, MA manual acupuncture, SA sham acupuncture

The demographic characteristics and clinical outcomes at baseline are those presented in Table 1. Missing data for baseline characteristics will not be imputed. Differences among the treatment groups at baseline will not be statistically tested.

\section{Analysis of the primary outcome}

In the analysis of the primary outcome, the response rates of the three groups at 8 weeks will be calculated and the $Z$-test for comparisons of proportions will be used with the full analysis set. Any missing data at 8 weeks will be imputed using the baseline value. There will be two comparisons. The first comparison is that between the EA group and the SA group. The second is that between the MA group and the SA group. The significance level will be adjusted at 0.025 for multiple comparisons using the Bonferroni method.

\section{Analysis of secondary outcomes}

For the NRS score, comparisons among the three groups will be assessed by a mixed-effect model with repeated measurement (MMRM) analysis using the NRS score at all follow-up time points as the dependent variable, treatment as the main factor, and the baseline value as a covariate. We set the model as

\section{$y_{i j}=\alpha+u_{i}+\beta_{1}$ time $_{i j}+\beta_{2}$ treat $_{i}+\varepsilon_{i j}$}

where $\alpha$ is the overall average and $u_{i}$ is an unknown random effect represented as a subject-specified effect. $\beta_{1}$ and $\beta_{2}$ are unknown fixed effects represented as time and treatment effects, respectively. The covariance matrix $G$ is unstructured, and $u_{i} \sim N(0, G)$. The random error $\varepsilon_{i j} \sim N\left(0, R_{i}\right)$. 
Table 1 Baseline characteristics

\begin{tabular}{lll}
\hline Baseline characteristic & Type & Levels or scale \\
\hline Gender & Categorical & Male or female \\
Age & Continuous & Years \\
Nationality & Categorical & Han or other \\
Duration of disease & Continuous & Years \\
Kellgren-Lawrence grade & Categorical & Grade II or III \\
Body mass index & Continuous & $\mathrm{kg} / \mathrm{m}^{2}$ \\
Years of education & Categorical & $<9,9-12$, or $>12$ \\
Affected knee & Categorical & Unilateral or bilateral \\
Previous treatments & Categorical & Injections, medication, physical therapy, acupuncture, exercise, etc. \\
Concomitant diseases & Categorical & Hypertension, coronary heart disease, diabetes mellitus, hyperlipidemia, etc. \\
Numerical rating scale & Continuous & Point \\
WOMAC pain subscale & Continuous & Point \\
WOMAC function subscale & Continuous & Point \\
WOMAC stiffness subscale & Continuous & Point \\
Physical health, SF-12 & Continuous & Point \\
Mental health, SF-12 & Continuous & .
\end{tabular}

WOMAC Western Ontario and McMaster Universities osteoarthritis index, SF-12 12-item Short Form Health Survey

The MMRM for secondary outcomes will be handled by PROC MIXED (SAS). The estimators of unknown parameters will be calculated using an expectation maximalization algorithm, which we expect will converge for the 480 participants in three groups and a single random intercept. If non-convergence does happen, we will consider correcting the initial value, changing the random effect, or using another analysis method like generalized estimating equations. Also, we will test the estimators or models based on a likelihood test and Bayesian information criterion methods.

The modified MMRM also has the center effect and time $\times$ treatment effect and it will be used in the sensitivity analysis. The modified MMRM is as follows:

$$
\begin{aligned}
y_{i j}= & \alpha+u_{i}+\beta_{1} \text { time }_{i j}+\beta_{2} \text { treat }_{i}+\beta_{3} \text { time }_{i j} \times \text { treat }_{i} \\
& +\beta_{4} \text { center }_{i}+\varepsilon_{i j}
\end{aligned}
$$

where $\alpha, \mathrm{u}_{i}, \beta_{1}, \beta_{2}$, and $\varepsilon_{i j}$ are defined as above. $\beta_{3}$ and $\beta_{4}$ are unknown fixed effects.

The same approach will be used to analyze the pain, function, and stiffness WOMAC subscales, and SF-12. If normality is violated in the continuous variables, a transformation will be performed before the comparison. A chi-square test will be used for the patient global assessment. These outcomes are shown in Table 2.

\section{Safety analyses}

Based on the potential relationship between acupuncture and adverse events, adverse events are categorized as treatment-related or not. Acupuncture-related adverse events will be summarized by group and compared using a chi-square test (or Fisher's exact test).

\section{Blinding analyses}

A kappa analysis will be used to determine whether participants correctly guessed their group assignment at a higher rate than would be expected by chance.

\section{Additional analyses}

Another three schemes to deal with any missing data for the primary outcome will be carried out to examine the robustness of the conclusion. First, any data missing at 8 weeks will be imputed using the last observation carried forward approach. Second, we will directly remove any missing data. Third, any data missing at 8 weeks will be imputed using multiple imputation [28]. By assuming the data missing are random, the missing data will be imputed using the Monte Carlo Markov chain method for multiple imputation with Proc MI (SAS). The initial seed will be set at 1000 and five datasets will be imputed. Any data missing for the primary outcome will be imputed by the observation value of age, gender, body mass index, Kellgren-Lawrence grade, and duration of disease. A subgroup analysis based on Kellgren-Lawrence grade will be performed.

\section{Discussion and trial status}

The trial will provide high-quality evidence of the efficacy of EA and MA for KOA. This paper provides details of the planned statistical analyses for the current 
Table 2 Primary and secondary outcomes

\begin{tabular}{|c|c|c|c|c|c|c|c|c|c|c|}
\hline \multirow[t]{3}{*}{ Outcomes } & \multirow[t]{3}{*}{$\mathrm{EA}(n)$} & \multirow[t]{3}{*}{ MA $(n)$} & \multirow[t]{3}{*}{$\operatorname{SAb}(n)$} & \multirow{3}{*}{$\begin{array}{l}P \\
\text { value }\end{array}$} & \multicolumn{6}{|l|}{ Pairwise comparison } \\
\hline & & & & & \multicolumn{2}{|l|}{ EA vs. SA } & \multicolumn{2}{|l|}{ MA vs. SA } & \multicolumn{2}{|l|}{ EA vs. MA } \\
\hline & & & & & Difference $(95 \% \mathrm{Cl})$ & $P$ value & Difference (95\% Cl) & $P$ value & Difference (95\% Cl) & $P$ value \\
\hline \multicolumn{11}{|c|}{ Success rate, no. (\%) } \\
\hline 4 weeks & $x(x x . x)$ & $x(x x . x)$ & $x(x x . x)$ & - & $x X . x(x X . X-X X . X)$ & $x x \cdot x$ & $x x . x(x x \cdot x-x x . x)$ & $x x \cdot x$ & - & - \\
\hline 8 weeks & $x(x x . x)$ & $x(x x . x)$ & $x(x x . x)$ & - & $x x . x(x x . x-x x . x)$ & $x x . x$ & $x x . x(x x . x-x x . x)$ & $x x . x$ & - & - \\
\hline 16 weeks & $x(x x . x)$ & $x(x x . x)$ & $x(x x . x)$ & - & $x x . x(x X . x-x x . x)$ & $x x . x$ & $X X . x(X X . X-X X . x)$ & $x x . x$ & - & - \\
\hline 26 weeks & $x(x x . x)$ & $x(x x . x)$ & $x(x x . x)$ & - & $x x \cdot x(x x \cdot x-x x \cdot x)$ & $x x . x$ & $x x \cdot x(x x \cdot x-x x \cdot x)$ & $x x . x$ & - & - \\
\hline \multicolumn{11}{|c|}{ Numerical Rating Scale, mean (SD) } \\
\hline Baseline & $x x . x(x x . x)$ & $x x . x(x x . x)$ & $x x . x(x x . x)$ & $x x \cdot x$ & $x x . x(x x . x-x x . x)$ & $x x . x$ & $x x . x(x x . x-x x . x)$ & $x x . x$ & $x x . x(x x . x-x x . x)$ & $x x . x$ \\
\hline 4 weeks & $x x . x(x x . x)$ & $x x . x(x x . x)$ & $x x . x(x x . x)$ & & $x x . x(x X . X-X X . X)$ & & $x x . x(x X . X-X X . x)$ & & $x x . x(x X . x-X X . x)$ & \\
\hline 8 weeks & $x x . x(x x . x)$ & $x x . x(x x . x)$ & $x x . x(x x . x)$ & & $x x . x(x x . x-x x . x)$ & & $x x . x(x x . x-x x . x)$ & & $x X . x(x X . X-X X . X)$ & \\
\hline 16 weeks & $x x . x(x x . x)$ & $x x . x(x x . x)$ & $x x . x(x x . x)$ & & $x x . x(x X . X-X X . x)$ & & $x X . X(x X . X-X X . X)$ & & $x x . x(x X . x-x x . x)$ & \\
\hline 26 weeks & $x x . x(x x . x)$ & $x x . x(x x . x)$ & $x x . x(x x . x)$ & & $X X . x(x X . X-X X . X)$ & & $X X . x(X X . X-X X . X)$ & & $x X . x(x X . X-X X . X)$ & \\
\hline \multicolumn{11}{|c|}{ WOMAC pain subscale, mean (SD) } \\
\hline Baseline & $x x . x(x x . x)$ & $x x . x(x x . x)$ & $x x . x(x x . x)$ & $x x \cdot x$ & $x x . x(x x . x-x x . x)$ & $x x . x$ & $X X . X(X X . X-X X . X)$ & $x x \cdot x$ & $X X . X(X X . X-X X . X)$ & $x x . x$ \\
\hline 4 weeks & $x x . x(x x . x)$ & $x x . x(x x . x)$ & $x x . x(x x . x)$ & & $x X . x(x X . X-X X . X)$ & & $X X . x(x X . X-X X . X)$ & & $X X . x(x X . X-X X . X)$ & \\
\hline 8 weeks & $x x . x(x x . x)$ & $x x . x(x x . x)$ & $x x . x(x x . x)$ & & $x x . x(x x . x-x x . x)$ & & $x x . x(x X . x-x x . x)$ & & $x x . x(x x . x-x x . x)$ & \\
\hline 16 weeks & $x x . x(x x . x)$ & $x x . x(x x . x)$ & $x x . x(x x . x)$ & & $x x . x(x X . x-x x . x)$ & & $x X . x(x X . x-x X . X)$ & & $x x . x(x X . x-x x . x)$ & \\
\hline 26 weeks & $x x . x(x x . x)$ & $x x . x(x x . x)$ & $x x . x(x x . x)$ & & $x x . x(x x . x-x x . x)$ & & $x x . x(x x \cdot x-x x \cdot x)$ & & $x x . x(x x . x-x x . x)$ & \\
\hline \multicolumn{11}{|c|}{ WOMAC function subscale, mean (SD) } \\
\hline Baseline & $x x . x(x x . x)$ & $x x . x(x X . x)$ & $x x . x(x x . x)$ & $x x \cdot x$ & $x x . x(x x . x-x x . x)$ & $x x . x$ & $x x . x(x x \cdot x-x x . x)$ & $x x \cdot x$ & $X X . x(X X . X-X X . X)$ & $x x . x$ \\
\hline 4 weeks & $x x . x(x x . x)$ & $x x . x(x x . x)$ & $x x . x(x x . x)$ & & $x x . x(x X . x-x x . x)$ & & $x x . x(x X . x-x x . x)$ & & $x x . x(x x . x-x x . x)$ & \\
\hline 8 weeks & $x x . x(x x . x)$ & $x x . x(x x . x)$ & $x x . x(x x . x)$ & & $x x . x(x x . x-x x . x)$ & & $x x . x(x x . x-x x . x)$ & & $x X . x(x X . X-X X . X)$ & \\
\hline 16 weeks & $x x . x(x x . x)$ & $x x . x(x x . x)$ & $x x . x(x x . x)$ & & $x x . x(x x . x-x x . x)$ & & $x x . x(x x . x-x x . x)$ & & $x x . x(x X . X-X X . X)$ & \\
\hline 26 weeks & $x x . x(x x . x)$ & $x x . x(x x . x)$ & $x x . x(x x . x)$ & & $X X . X(X X . X-X X . X)$ & & $X X . X(X X . X-X X . X)$ & & $X X . X(X X . X-X X . X)$ & \\
\hline \multicolumn{11}{|c|}{ WOMAC stiffness subscale, mean (SD) } \\
\hline Baseline & $x x . x(x x . x)$ & $x x . x(x x . x)$ & $X X . x(x X . X)$ & $x x \cdot x$ & $X X . X(X X . X-X X . X)$ & $x x . x$ & $X X . X(X X . X-X X . X)$ & $x x \cdot x$ & $X X . X(X X . X-X X . X)$ & $x x . x$ \\
\hline 4 weeks & $x X . X(x X . X)$ & $X X . X(X X . X)$ & $X X . X(X X . X)$ & & $X X . X(X X . X-X X . X)$ & & $X X . X(X X . X-X X . X)$ & & $X X . X(X X . X-X X . X)$ & \\
\hline 8 weeks & $x X . x(x x . x)$ & $X X . X(X X . X)$ & $X X . X(X X . X)$ & & $X X . X(X X . X-X X . X)$ & & $X X . X(X X . X-X X . X)$ & & $X X . X(X X . X-X X . X)$ & \\
\hline 16 weeks & $x x . x(x x . x)$ & $x x . x(x x . x)$ & $x x . x(x x . x)$ & & $x X . x(x X . X-X x . x)$ & & $x x . x(x X . x-X x . x)$ & & $x x . x(x X . X-X X . X)$ & \\
\hline 26 weeks & $x x . x(x x . x)$ & $x x . x(x x . x)$ & $x x . x(x x . x)$ & & $X X . x(X X . X-X x \cdot x)$ & & $X X . x(x X . X-X x . x)$ & & $x x . x(x X . x-x X . X)$ & \\
\hline \multicolumn{11}{|c|}{ Patient global assessment, mean (SD) } \\
\hline Baseline & $x x . x(x x . x)$ & $x x . x(x x . x)$ & $x x . x(x x . x)$ & $x x \cdot x$ & $x x . x(x x . x-x x . x)$ & $x x . x$ & $x x . x(x X . x-x x . x)$ & $x x . x$ & $x x . x(x x . x-x x . x)$ & $x x . x$ \\
\hline 4 weeks & $x x . x(x x . x)$ & $x x . x(x x . x)$ & $x x . x(x x . x)$ & & $x x . x(x x . x-x x . x)$ & & $x x . x(x x . x-x x . x)$ & & $x x . x(x x . x-x x . x)$ & \\
\hline 8 weeks & $x x . x(x x . x)$ & $x x . x(x X . x)$ & $x x . x(x x . x)$ & & $x x . x(x x . x-x x . x)$ & & $X X . x(x X . X-X X . X)$ & & $x x . x(x X . x-x x . x)$ & \\
\hline 16 weeks & $x x . x(x x . x)$ & $x x . x(x x . x)$ & $x x . x(x x . x)$ & & $x x . x(x x . x-x x . x)$ & & $x x . x(x x . x-x x . x)$ & & $x x . x(x x . x-x x . x)$ & \\
\hline 26 weeks & $x X . x(x x . x)$ & $x X . x(x x . x)$ & $x x . x(x x . x)$ & & $X X . X(X X . X-X X . X)$ & & $X X . X(X X . X-X X . X)$ & & $X X . X(X X . X-X X . X)$ & \\
\hline \multicolumn{11}{|c|}{ SF-12 physical health, mean (SD) } \\
\hline Baseline & $X X . x(x X . x)$ & $X X . x(x X . X)$ & $X X . x(x X . X)$ & $x x \cdot x$ & $X X . X(X X . X-X X . X)$ & $x x . x$ & $X X . X(X X . X-X X . X)$ & $x x . x$ & $X X . X(X X . X-X X . X)$ & $x x . x$ \\
\hline 4 weeks & $x x . x(x x . x)$ & $x x . x(x x . x)$ & $x x . x(x x . x)$ & & $x X . x(x X . X-X X . X)$ & & $x x . x(x X . X-X X . X)$ & & $x x . x(x x . x-x x . x)$ & \\
\hline 8 weeks & $x x . x(x X . X)$ & $x x . x(x X . X)$ & $X X . x(x X . X)$ & & $X X . X(X X . X-X X . X)$ & & $X X . X(X X . X-X X . X)$ & & $X X . X(X X . X-X X . X)$ & \\
\hline 16 weeks & $x x . x(x x . x)$ & $x x . x(x x . x)$ & $x x . x(x x . x)$ & & $x x . x(x X . X-x x . x)$ & & $x x . x(x x . x-x x . x)$ & & $x X . x(x X . X-X X . X)$ & \\
\hline 26 weeks & $x X . x(x X . X)$ & $X X . x(x X . X)$ & $x x . x(x x . x)$ & & $x x . x(x X . X-x x . x)$ & & $x x . x(x X . X-x x . x)$ & & $X X . X(X X . X-X X . X)$ & \\
\hline
\end{tabular}


Table 2 Primary and secondary outcomes (Continued)

\begin{tabular}{|c|c|c|c|c|c|c|c|c|c|c|}
\hline \multirow[t]{3}{*}{ Outcomes } & \multirow[t]{3}{*}{$\mathrm{EA}(n)$} & \multirow[t]{3}{*}{$\mathrm{MA}(n)$} & \multirow[t]{3}{*}{ SAb (n) } & \multirow{3}{*}{$\begin{array}{l}P \\
\text { value }\end{array}$} & \multicolumn{6}{|l|}{ Pairwise comparison } \\
\hline & & & & & \multicolumn{2}{|l|}{ EA vs. SA } & \multicolumn{2}{|l|}{ MA vs. SA } & \multicolumn{2}{|l|}{ EA vs. MA } \\
\hline & & & & & Difference (95\% Cl) & $P$ value & Difference (95\% Cl) & $P$ value & Difference $(95 \% \mathrm{Cl})$ & $P$ value \\
\hline Baseline & $x x . x(x x . x)$ & $x x . x(x x . x)$ & $x x . x(x x . x)$ & $x x . x$ & $x x \cdot x(x x \cdot x-x x \cdot x)$ & $x x \cdot x$ & $X X . X(X X . X-X X . x)$ & $x x \cdot x$ & $x X . x(x X . X-X X . x)$ & $x x . x$ \\
\hline 4 weeks & $x x . x(x x . x)$ & $x x . x(x x . x)$ & $x x . x(x x . x)$ & & $x X . x(x X . X-X X . x)$ & & $X X . X(X X . X-X X . X)$ & & $X X . X(X X . X-X X . X)$ & \\
\hline 8 weeks & $x x . x(x x . x)$ & $x x . x(x x . x)$ & $x x . x(x x . x)$ & & $x x . x(x x . x-x x . x)$ & & $x x . x(x x . x-x X . x)$ & & $x x . x(x x . x-x x . x)$ & \\
\hline 16 weeks & $x X . X(X X . X)$ & $x X . x(x X . x)$ & $X X . X(X X . X)$ & & $x X . x(x X . X-x X . x)$ & & XX.X (XX.X-XX.X) & & XX.X (XX.X-XX.X) & \\
\hline 26 weeks & $x x . x(x x . x)$ & $x x . x(x x . x)$ & $x x . x(x x . x)$ & & $X X . x(X X . X-X X . X)$ & & $x x . x(x x . x-x x . x)$ & & $x x . x(x x . x-x x . x)$ & \\
\hline
\end{tabular}

EA electro-acupuncture, MA manual acupuncture, SA sham acupuncture, WOMAC Western Ontario and McMaster Universities Osteoarthritis Index, SD standard deviation, SF-12 12-item Short Form Health Survey

trial and will help to reduce the risks of outcome reporting bias and data-driven results [29]. This paper has been prepared in accordance with the published guidelines for the content of statistical analysis plans [30]. As of October 2018, 480 patients from nine centers had been randomized. The final date of follow-up is April 7, 2019. This analysis plan was written prior to completion of the trial data collection phase.

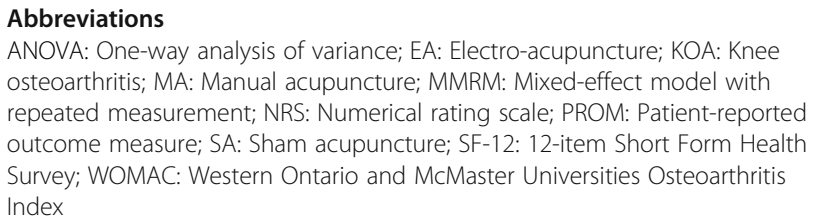

ANOVA: One-way analysis of variance; EA: Electro-acupuncture; KOA: Knee osteoarthritis; MA: Manual acupuncture; MMRM: Mixed-effect model with repeated measurement; NRS: Numerical rating scale; PROM: Patient-reported outcome measure; SA: Sham acupuncture; SF-12: 12-item Short Form Health Survey; WOMAC: Western Ontario and McMaster Universities Osteoarthritis Index

\section{Acknowledgements}

None.

\section{Authors' contributions}

JFT, JWY, ZSY, and CZL proposed the statistical analysis plan. JFT and YW drafted the manuscript. CZL led the trial concept and assembled the study group. All authors reviewed and approved the final manuscript.

\section{Funding}

The trial is funded by the Beijing Municipal Science \& Technology Commission (D171100003217003) and the Special Funding for Clinical Medicine Development of the Beijing Municipal Hospitals Administration (XMLX201607). The funders have no role in study design, data collection, analysis and interpretation, decision to publish, or preparation of the manuscript.

\section{Availability of data and materials}

No original data are currently available relating to the detailed statistical analysis plan. The datasets used during the current study will be available from the corresponding author on reasonable request.

\section{Ethics approval and consent to participate}

Ethics approval was granted by the research ethics committee of Beijing Hospital of Traditional Chinese Medicine Affiliated to Capital Medical University (2017BL-077-01) and at each participating site. Written informed consent will be obtained from each participant prior to enrolment in the study.

\section{Competing interests}

The authors declare that they have no competing interests.

\section{Author details}

${ }^{1}$ School of Acupuncture-Moxibustion and Tuina, Beijing University of Chinese Medicine, 11 Beisanhuan East Road, Chaoyang District, Beijing 100029, China. ${ }^{2}$ Department of Statistics, School of Mathematical Sciences and SJTU-Yale
Joint Center for Biostatistics, Shanghai Jiao Tong University, Minhang District, Shanghai, China. ${ }^{3}$ Department of Bioinformatics and Biostatistics, School of Life Sciences and Biotechnology and SJTU-Yale Joint Center for Biostatistics, Shanghai Jiao Tong University, Minhang District, Shanghai, China.

${ }^{4}$ Department of Statistics, School of Mathematical Sciences, Shanghai Jiao Tong University, Minhang District, Shanghai, China.

Received: 15 March 2019 Accepted: 10 June 2019

Published online: 04 July 2019

\section{References}

1. Glyn-Jones S, Palmer AJ, Agricola R, Price AJ, Vincent TL, Weinans H, et al. Osteoarthritis. Lancet. 2015:386:376-87.

2. Tang X, Wang S, Zhan S, et al. The Prevalence of Symptomatic Knee Osteoarthritis in China: Results From the China Health and Retirement Longitudinal Study. Arthritis Rheumatol. 2016;68:648-53.

3. Guillemin F, Rat AC, Mazieres B, et al. Prevalence of symptomatic hip and knee osteoarthritis: a two-phase population-based survey. Osteoarthr Cartil. 2011;19:1314-22.

4. Hiligsmann M, Cooper C, Arden N, Boers M, Branco JC, Luisa Brandi M, et al. Health economics in the field of osteoarthritis: an expert's consensus paper from the European Society for Clinical and Economic Aspects of Osteoporosis and Osteoarthritis (ESCEO). Semin Arthritis Rheum. 2013:43:303-13.

5. Mahler E, Minten MJ, Leseman-Hoogenboom MM, Poortmans P, Leer J, Boks SS, et al. Effectiveness of low-dose radiation therapy on symptoms in patients with knee osteoarthritis: a randomised, double-blinded, shamcontrolled trial. Ann Rheum Dis. 2019;78:83-90.

6. da Costa BR, Reichenbach S, Keller N, Nartey L, Wandel S, Jüni P, et al. Effectiveness of non-steroidal anti-inflammatory drugs for the treatment of pain in knee and hip osteoarthritis: a network meta-analysis. Lancet. 2017; 390:e21-21e33.

7. McAlindon TE, Bannuru RR, Sullivan MC, et al. OARSI guidelines for the nonsurgical management of knee osteoarthritis. Osteoarthr Cartil. 2014;22:363-88.

8. Jones CA, Beaupre LA, Johnston DW, Suarez-Almazor ME. Total joint arthroplasties: current concepts of patient outcomes after surgery. Rheum Dis Clin N Am. 2007:33:71-86.

9. Su D, Li L. Trends in the use of complementary and alternative medicine in the United States: 2002-2007. J Health Care Poor Underserved. 2011;22:296-310.

10. Vickers AJ, Cronin AM, Maschino AC, et al. Acupuncture for chronic pain: individual patient data meta-analysis. Arch Intern Med. 2012;172:1444-53.

11. Hinman RS, McCrory P, Pirotta M, et al. Acupuncture for chronic knee pain: a randomized clinical trial. JAMA. 2014;312:1313-22.

12. White $A$, Cummings $M$, Barlas $P$, et al. Defining an adequate dose of acupuncture using a neurophysiological approach--a narrative review of the literature. Acupunct Med. 2008;26:111-20.

13. Zhang Q, Yue J, Lu Y. Acupuncture treatment for chronic knee pain: study by Hinman et al underestimates acupuncture efficacy. Acupunct Med. 2015:33:170

14. Armour M, Smith CA. Treating primary dysmenorrhoea with acupuncture: a narrative review of the relationship between acupuncture 'dose' and menstrual pain outcomes. Acupunct Med. 2016;34:416-24. 
15. He W, Zhu B, Yu X, Liu B, Xu N, Jing X. Compairison between western and Chinese acupuncture and its enlightenment. Zhongguo Zhen Jiu. 2015;35:105-8.

16. Lin LL, Li YT, Tu JF, Yang JW, Sun N, Zhang S, et al. Effectiveness and feasibility of acupuncture for knee osteoarthritis: a pilot randomized controlled trial. Clin Rehabil. 2018;32:1666-75.

17. Noordergraaf A, Silage D. Electroacupuncture. IEEE Trans Biomed Eng. 1973;20:364-6.

18. Tu JF, Yang JW, Lin LL, Wang TQ, Du YZ, Liu ZS, et al. Efficacy of electroacupuncture and manual acupuncture versus sham acupuncture for knee osteoarthritis: study protocol for a randomised controlled trial. Trials. 2019;20:79.

19. Hochberg MC, Altman RD, Brandt KD, et al. Guidelines for the medical management of osteoarthritis. Part II. Osteoarthritis of the knee. American College of Rheumatology. Arthritis Rheum. 1995;38:1541-6.

20. Forestier R, Desfour $\mathrm{H}$, Tessier JM, et al. Spa therapy in the treatment of knee osteoarthritis: a large randomised multicentre trial. Ann Rheum Dis. 2010;69:660-5

21. Hawker GA, Mian S, Kendzerska T, et al. Measures of adult pain: Visual Analog Scale for Pain (VAS Pain), Numeric Rating Scale for Pain (NRS Pain), McGill Pain Questionnaire (MPQ), Short-Form McGill Pain Questionnaire (SFMPQ), Chronic Pain Grade Scale (CPGS), Short Form-36 Bodily Pain Scale (SF-36 BPS), and Measure of Intermittent and Constant Osteoarthritis Pain (ICOAP). Arthritis Care Res (Hoboken). 2011:63(Suppl 11):S240-52.

22. Tubach F, Ravaud P, Baron G, et al. Evaluation of clinically relevant changes in patient reported outcomes in knee and hip osteoarthritis: the minimal clinically important improvement. Ann Rheum Dis. 2005;64:29-33.

23. Bellamy N, Buchanan WW, Goldsmith $\mathrm{CH}$, et al. Validation study of WOMAC: a health status instrument for measuring clinically important patient relevant outcomes to antirheumatic drug therapy in patients with osteoarthritis of the hip or knee. J Rheumatol. 1988;15:1833-40.

24. Berman BM, Lao L, Langenberg P, et al. Effectiveness of acupuncture as adjunctive therapy in osteoarthritis of the knee: a randomized, controlled trial. Ann Intern Med. 2004;141:901-10.

25. Ware J, Kosinski M, Keller SD. A 12-Item Short-Form Health Survey: construction of scales and preliminary tests of reliability and validity. Med Care. 1996;34:220-33.

26. Devilly GJ, Borkovec TD. Psychometric properties of the credibility/ expectancy questionnaire. J Behav Ther Exp Psychiatry. 2000:31:73-86.

27. Schulz KF, Altman DG, Moher D. CONSORT 2010 statement: updated guidelines for reporting parallel group randomized trials. Ann Intern Med. 2010;152:726-32

28. Li P, Stuart EA, Allison DB. Multiple Imputation: A Flexible Tool for Handling Missing Data. JAMA. 2015;314:1966-7.

29. Finfer S, Bellomo R. Why publish statistical analysis plans. Crit Care Resusc. 2009;11:5-6.

30. Gamble C, Krishan A, Stocken D, Lewis S, Juszczak E, Doré C, et al. Guidelines for the Content of Statistical Analysis Plans in Clinical Trials. JAMA. 2017;318:2337-43.

\section{Publisher's Note}

Springer Nature remains neutral with regard to jurisdictional claims in published maps and institutional affiliations.

Ready to submit your research? Choose BMC and benefit from:

- fast, convenient online submission

- thorough peer review by experienced researchers in your field

- rapid publication on acceptance

- support for research data, including large and complex data types

- gold Open Access which fosters wider collaboration and increased citations

- maximum visibility for your research: over $100 \mathrm{M}$ website views per year

At $\mathrm{BMC}$, research is always in progress.

Learn more biomedcentral.com/submissions 